\title{
Preexposure of the conditioning context and latent inhibition from reduced conditioning
}

\author{
DENNIS C. WRIGHT and KAREN K. GUSTAVSON \\ University of Missouri-Columbia, Columbia, Missouri
}

\begin{abstract}
Recently, Wright, Skala, and Peuser (1986) reported a failure to find latent inhibition in rats given unreinforced preconditioning exposure to a tone (PCE) when PCE and conditioning (toneshock) occurred in the same apparatus/context and testing for lick suppression occurred in a different context. The no-PCE control group used for comparison had not been exposed to the tone or the conditioning context prior to conditioning. In the present experiment, the no-PCE control/comparison group received preexposure to the conditioning context, but not to the tone. Although the three groups given 12, 28, or 60 PCEs in the conditioning context did not differ from each other, they all showed less lick suppression in the test context than the no-PCE control group.
\end{abstract}

Lubow, Rifkin, and Alek (1976) and Channell and Hall (1981) demonstrated that preexposure to stimuli that were later to be used in a discrimination learning paradigm either retarded or facilitated acquisition of the discrimination, depending on the context in which preexposure had occurred. Lubow et al. (1976) showed that rats that had been preexposed to the training stimulus in the training context, and thus encountered an "old" stimulus in an "old" context $\left(\mathrm{S}_{\mathrm{o}} \mathrm{C}_{\mathrm{o}}\right)$ during training, were retarded in acquisition relative to animals that had not been preexposed to the training stimulus or context. These nonpreexposed animals, for whom both the stimulus and context were "new" $\left(S_{n} C_{n}\right)$, showed retarded acquisition relative to animals that had been preexposed to the training context but not to the stimulus $\left(\mathrm{S}_{\mathrm{n}} \mathrm{C}_{\mathrm{o}}\right)$. Although statistically reliable, the difference between $S_{o} C_{o}$ and $S_{n} C_{n}$ animals (Lubow et al., 1976, Figure 2) does not appear to be as large as the difference between either of those groups and $\mathrm{S}_{\mathrm{n}} \mathrm{C}_{\mathrm{o}}$ animals. Grant and Young (1971) preexposed mice to a tone $C S$ in a one-way shuttle box $\left(S_{0} C_{0}\right)$, to the apparatus only $\left(\mathrm{S}_{\mathrm{n}} \mathrm{C}_{\mathrm{o}}\right)$, or to neither the apparatus nor the CS $\left(S_{n} C_{n}\right)$. They demonstrated that the $S_{n} C_{o}$ group later acquired an avoidance response more rapidly than either the $S_{n} C_{n}$ or $S_{o} C_{o}$ group but that the $S_{n} C_{n}$ and $S_{o} C_{o}$ groups did not differ from each other.

Recently, Wright, Skala, and Peuser (1986) reported a failure to demonstrate latent inhibition (LI) in rats given preconditioning exposure to a tone stimulus (PCE). In their experiments, the no-preexposure comparison group entered conditioning without having experienced either the tone CS or the conditioning context and, therefore, might be considered to be a $S_{n} C_{n}$ group. The rats given PCE in the conditioning context had experienced both the $\mathrm{CS}$ and the conditioning context prior to conditioning and

We thank Donald H. Kausler and Ruth E. Wright for reviewing this manuscript and providing valuable comments.

Requests for reprints should be sent to Dennis C. Wright, Department of Psychology, University of Missouri, Columbia, MO 65211. can be considered to be a $\mathrm{S}_{\mathrm{o}} \mathrm{C}_{\mathrm{o}}$ group during conditioning. Wright et al. may have failed to demonstrate LI because demonstrating a $\mathrm{S}_{n} \mathrm{C}_{n}-\mathrm{S}_{0} \mathrm{C}_{\mathrm{o}}$ difference may require more extensive preexposure to the stimulus and/or conditioning context than was afforded in their design. The present experiment was based on the assumption that a difference between $S_{n} C_{o}$ and $S_{o} C_{o}$ groups could be demonstrated with relatively limited CS and/or context preexposure.

All rats were lick trained in Apparatus A, given four 8-min conditioning context preexposure sessions in Apparatus X, conditioned in Apparatus X, and tested for lick suppression in Apparatus A. One group of rats received no CS presentations during preexposure, and thus were expected to be a $\mathrm{S}_{\mathrm{n}} \mathrm{C}_{\mathrm{o}}$ group during conditioning. The remaining three groups were intended to be $S_{0} C_{0}$ groups and received 3,7 , or 15 CS presentations during these sessions so we could attempt to measure differences that might occur among $\mathrm{S}_{\mathrm{o}} \mathrm{C}_{\mathrm{o}}$ groups as a function of PCE number.

\section{METHOD}

\section{Subjects}

Twenty-eight 276-347 g male Sprague-Dawley rats (Sasco) served as subjects. All rats were individually housed in mesh-bottom cages, with a 12/12 light/dark cycle, and ad-lib food. Access to water was provided for $1 \mathrm{~h}$ each day, immediately after the experimental session for that day. All experimental sessions were run during the 10-h time span between $1 \mathrm{~h}$ after lights on and $1 \mathrm{~h}$ before lights off.

\section{Apparatus}

Box A, the lick train/test chamber, was constructed of Plexiglas and had inside dimensions of $40.6 \times 20.3 \times 30.5 \mathrm{~cm}(1 \times \mathrm{w} \times \mathrm{h})$ and had a floor of $0.16-\mathrm{cm}$ mesh copper screen. A $0.64-\mathrm{cm}$-diameter hole that was centered in one $20.3-\mathrm{cm}$ end wall, $3.8 \mathrm{~cm}$ above the floor, permitted the lick tube to protrude $0.95 \mathrm{~cm}$ into the box. The lick tube was connected to a computer control system via a solid-state lickometer. The walls of Box A were painted with alternating 1.9-cm black and white stripes. Box X, the conditioning chamber, was $29.2 \times 22.2 \times 22.9 \mathrm{~cm}$ with three unpainted galvanized steel walls and a clear Plexiglas front. The shock grid floor was constructed of $0.32-\mathrm{cm}$ stainless steel rods mounted on $1.3-\mathrm{cm}$ centers. 
Both boxes had clear Plexiglas ceilings with an $8-\Omega, 1.2-\mathrm{cm}$ diameter speaker mounted at the center for delivery of an $86-\mathrm{dB}(\mathrm{A}), 3-\mathrm{kHz}$ sine wave tone CS. Both boxes were at different loci in the same room. The room was lighted with florescent fixtures in the ceiling of the room when Box $\mathrm{A}$ was in use. The ambient noise level in Box A was less than $60 \mathrm{~dB}(\mathrm{~A})$. Box $X$ was placed inside a sound-attenuation chamber with an exhaust fan that produced an ambient noise level of $66 \mathrm{~dB}(\mathrm{~A})$ within Box X and had a $25-W$ incandescent bulb centered $40.6 \mathrm{~cm}$ over the clear ceiling of Box X.

\section{Procedure}

The rats were acclimated to the light/dark cycle for 1 month and the 23-h water-deprivation schedule for 2 weeks prior to the start of training. After 7 days of handling, lick training, PCE, conditioning, and lick suppression testing, sessions were administered over 10 consecutive days as follows:

Days 1-4, lick training in Box A: During each daily session, the rats were allowed to lick for water for $300 \mathrm{sec}$ after the completion of the first 100 licks. The time required to complete the first 100 licks was recorded. Time to Lick 100 on Training Day 4 was used to assign 7 rats to each of four matched groups.

Days 5-8, PCE in Box X: During each daily 480-sec session, the rats in Group 12 were presented three 10-sec CSs delivered on a VT 140sec schedule, those in Group 28 received 7 CSs (VT 60-sec), those in Group 60 received 15 CSs (VT 28-sec), and the rats in Group 0 received no CS presentations.

Day 9, conditioning in Box X: All rats received 2 tone-shock pairings (10-sec CS followed immediately by $1-\mathrm{sec}, 1-\mathrm{mA}$ scrambled footshock) during a single 480 -sec session in Box X. The first pairing was delivered $120 \mathrm{sec}$, and the second $360 \mathrm{sec}$, after the start of the session.

Day 10, test in Box A: All rats received a single test for conditioned lick suppression in Box A. At the completion of Lick 100, the tone CS was turned on and remained on until the rat completed 10 additional licks or until $900 \mathrm{sec}$ had elapsed. The time from Lick 100 to Lick 110 (i.e., time to complete 10 licks in the presence of the CS) was recorded. Rats failing to complete those 10 licks were assigned a score of 900 , thus making nonparametric analyses appropriate for the test data. (Parametric ANOVAs and $t$ tests yielded an identical pattern of statistical significance.)

\section{Results and Discussion}

One rat (Group 28) failed to complete 100 licks during test and was eliminated from the analysis of results. A Kruskal-Wallis one-way analysis of variance revealed no group differences in time to complete the first 100 licks in test $[H(3)=0.69]$.

Three animals (all in Group 0) failed to complete 10 licks in the presence of the CS and were assigned scores of 900 . The group mean and median times to complete 10 licks in the presence of the CS are shown in Table 1. The Kruskal-Wallis analysis of time from Lick 100 to Lick 110 for all groups was significant $[H(3)=8.19$, $p<.05]$. As predicted, one-tailed Mann-Whitney tests revealed that Group 0 showed significantly more suppression than all other groups ( 0 vs. $12, U=7, p<.013$; 0 vs. $28, U=7, p<.026 ; 0$ vs. $60, U=5, p<.006)$.

Table 1

Median and Mean Seconds from Lick 100 to Lick 110

\begin{tabular}{ccc}
\hline Group & Median & Mean \\
\hline 0 & 600.27 & 637.09 \\
12 & 213.18 & 277.49 \\
28 & 182.71 & 297.43 \\
60 & 125.60 & 212.30 \\
\hline
\end{tabular}

Clearly, robust LI is demonstrated here with as few as 12 PCEs.

A Kruskal-Wallis analysis that included only Groups 12,28 , and 60 confirmed the impression that the groups given different amounts of PCE did not differ from one another $[H(2)=0.30]$. Relatively few studies of conditioned suppression in rats have directly compared groups given different amounts of PCE. Domjan and Siegel (1971) gave rats $0,2,5,10,25$, or 50 PCEs. Those rats given 25 and 50 PCEs did not appear to differ from each other but did show less conditioned suppression than the others. Thus, in their study, LI appears to reach an asymptote at between 10 and 25 PCEs. Lantz (1973) used 0, 10,30 , or 60 PCEs, with an apparent asymptote at between 10 and 30 PCEs. Lubow, Wagner, and Weiner (1982) showed an asymptote at between 20 and 40 PCEs using $0,20,40$, or 80 PCEs. Comparing across separate experiments, Wright et al. (1986) noted a failure to find differences between rats given 15 PCEs and rats given 30 PCEs in the conditioning context. Given previous research, failure to find a difference between groups given 12 PCEs and 60 PCEs in the current study is not surprising.

The robust $\mathrm{LI}$ demonstrated with as few as 12 PCEs in the present experiment confirms the assumption that a difference in conditioned lick suppression is relatively easy to demonstrate between rats that have and have not been preexposed to the CS, provided that all rats have been preexposed to the conditioning context. The present experiment, when considered in conjunction with the data of Wright et al. (1986), indicates that the presence or absence of preexposure to the conditioning context is as important a variable for demonstrations of $\mathrm{LI}$ as is the presence or absence of preexposure to the conditioning stimulus.

\section{REFERENCES}

Channell, S., \& Hall, G. (1981). Facilitation and retardation of discrimination learning after exposure to the stimuli. Journal of Experimental Psychology: Animal Behavior Processes, 7, 437-446.

DOMJAN, M., \& SIEGEL, S. (1971). Conditioned suppression following CS preexposure. Psychonomic Science, 25, 11-12.

Grant, M., \& Young, D. (1971). The effects of preexposure to a learning apparatus. Behavior Research Methods \& Instrumentation, 3, 251-252.

LANTZ, A. E. (1973). Effect of number of trials, interstimulus interval, and dishabituation during CS habituation on subsequent conditioning in a CER paradigm. Animal Learning \& Behavior, 1, 273-277.

Lubow, R. E., RIFKIN, B., \& ALEK, M. (1976). The context effect: The relationship between stimulus preexposure and environmental preexposure determines subsequent learning. Journal of Experimental Psychology: Animal Behavior Processes, 2, 38-47.

LUBOW, R. E., WAGNER, M., \& WeINER, I. (1982). The effects of compound stimulus preexposure of two elements differing in salience on the acquisition of conditioned suppression. Animal Learning \& Behavior, 10, 483-489.

Wright, D. C., Skala, K. D., \& Peuser, K. A. (1986). Latent inhibition from context-dependent retrieval of conflicting information. Bulletin of the Psychonomic Society, 24, 152-154.

(Manuscript received for publication June 5, 1986.) 